\title{
Un mort pour son chaudron
}

Ou comment faire du dieu-objet du palo monte un être irremplaçable (Cuba)

A dead for a caldron. Or how the god-object from palo monte becomes an irremplaceable entity (Cuba)

\section{Katerina Kerestetzi}

\section{OpenEdition}

\section{Journals}

Édition électronique

URL : https://journals.openedition.org/tc/6386

DOI : $10.4000 /$ tc. 6386

ISBN : $1952-420 X$

ISSN : 1952-420X

Éditeur

Éditions de l'EHESS

\section{Édition imprimée}

Date de publication : 15 juin 2012

Pagination : 48-65

ISBN : 2-7351-1512-7

ISSN : 0248-6016

\section{Référence électronique}

Katerina Kerestetzi, « Un mort pour son chaudron », Techniques \& Culture [En ligne], 58 | 2012, mis en ligne le 07 décembre 2012, consulté le 29 septembre 2022. URL : http://journals.openedition.org/tc/ 6386 ; DOI : https://doi.org/10.4000/tc.6386 


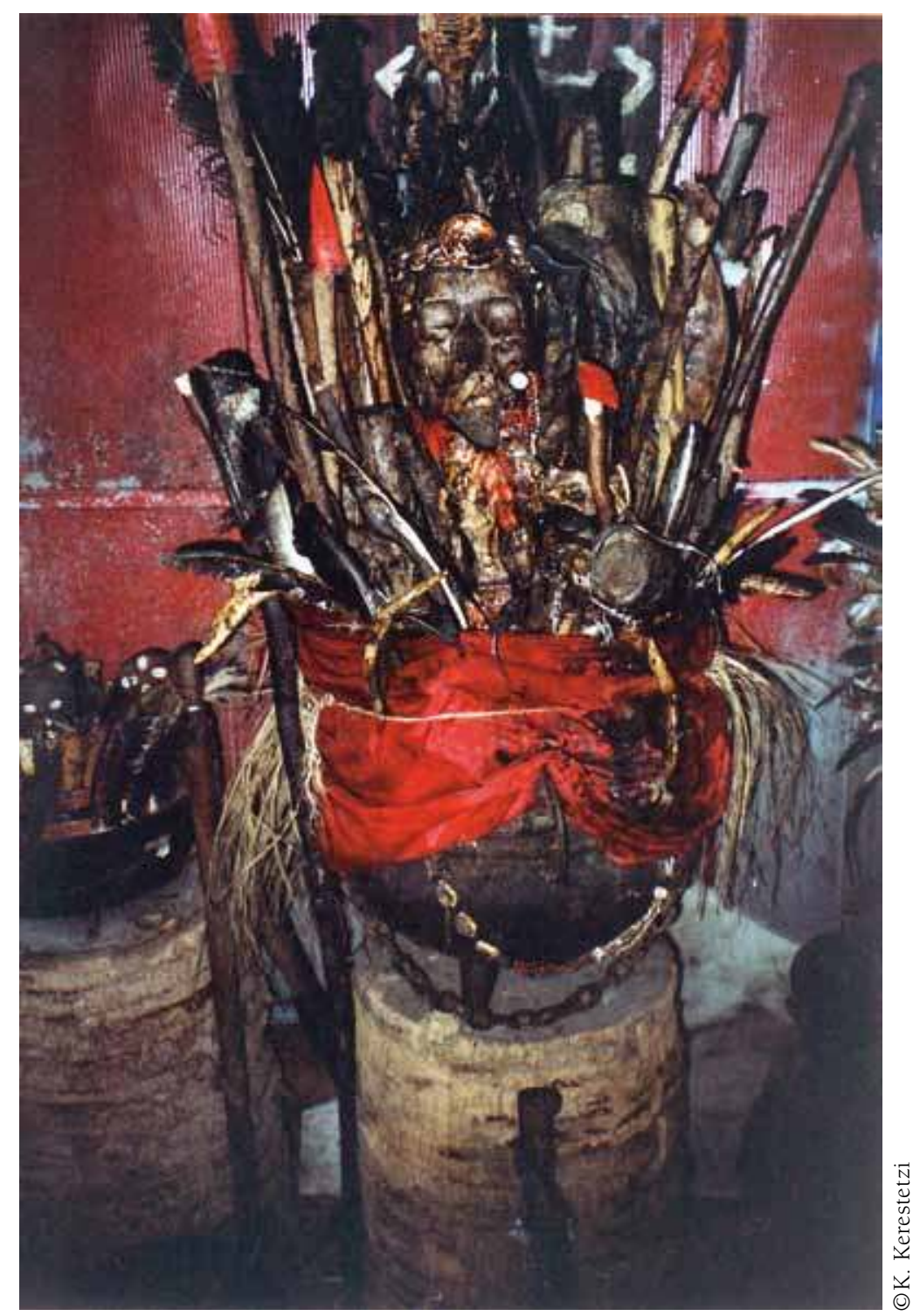




\section{UN MORT POUR SON CHAUDRON}

\section{OU COMMENT FAIRE DU DIEU-OBJET DU PALO MONTE UN ÊTRE IRREMPLAÇABLE (CUBA)}

Le palo monte, culte initiatique cubain d'origine bantoue, dont les pratiquants font remonter l'origine à l'ancien royaume Kongo, est présent dans toutes les régions de l'île de Cuba. Il n'existe pas de statistiques précises, mais sa pratique est en pleine expansion. C'est d'ailleurs le cas pour toutes les modalités religieuses en vigueur à Cuba. On assiste en effet au foisonnement des Églises évangéliques ou des Témoins de Jéhovah, qui se développent à côté du catholicisme avec le même rythme frénétique que les religions dites « afro-cubaines». Outre le palo monte, celles-ci incluent la santería (d'origine yoruba) et la société secrète masculine abakuá (d'origine efik-ibibio). La santería ou le culte abakuá ont largement suscité l'attention des chercheurs, dès le début du xxe siècle mais aussi plus récemment ${ }^{1}$. En revanche, les études exclusivement dédiées au palo monte sont plus rares $^{2}$, en partie parce qu'il demeure stigmatisé comme une pratique sorcière plutôt que religieuse dans le champ religieux local de même que dans le discours de certains chercheurs. C'est ce qui m'a conduite à m'y intéresser.

Précisons que comme dans les autres religions afro-cubaines, le parcours dans le palo monte est une lutte personnelle par laquelle on vise à améliorer les conditions matérielles de son quotidien, ses relations et sa santé, à accumuler de la force afin d'affronter ses ennemis ainsi que les vicissitudes de l'existence. Pour le faire, les pratiquants, les paleros, établissent un lien rituel avec certains esprits des morts, les nfumbis. Ils peuvent bénéficier ainsi de leur protection et des immenses pouvoirs dont ils les créditent (don d'ubiquité, prescience, contrôle sur les éléments de la nature). Pour créer ce lien, le palero doit conclure un pacte rituel avec un mort trouvé dans un cimetière. Une fois le pacte conclu, il doit déterrer le mort et récupérer certains de ses ossements (le crâne est la partie la 


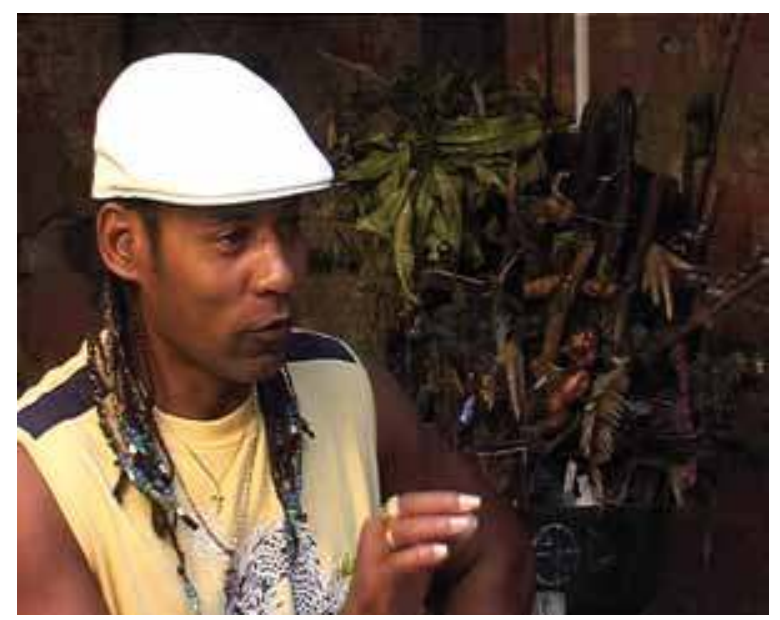

plus convoitée). Il apporte ensuite ces os dans son temple et les introduit dans un chaudron, la nganga, lequel devient ainsi le nouveau corps de l'esprit.

Ce corps hybride, qui inclut aussi une multitude d'éléments prélevés à la nature (minéraux, végétaux, restes d'animaux, etc.) et de métaux ouvragés (machettes, chaînes, menottes, fers à cheval), est l'objet central de la pratique du palo monte ${ }^{3}$ : aucun rituel, opération magique ou divination ne peut s'effectuer sans sa médiation (Figure 1). L'existence de ce chaudron est ainsi la condition préalable à l'accomplissement du culte; elle est un principe organisationnel de la pratique religieuse, son noyau dur : tout acte, toute décision et tout rituel se font en concertation et en coopération avec la nganga. Dans un contexte de variabilité extrême où chaque palero définit sa méthodologie religieuse de manière autonome et singulière, l'agentivité du chaudron, son rôle actif dans les processus rituels, est constitutive de la pratique religieuse.

On ne peut pas être palero sans nganga, sans ce chaudron qui est à la source de tous les pouvoirs des croyants. Les officiants déclarent que « sans nganga, il n'y a pas de palero, sans la nganga nous ne sommes rien".

Marque de fabrique des paleros dans le contexte religieux fortement varié de Cuba et objet de fixation identitaire, la nganga est le symbole du palo monte et de ses adeptes pour le reste de la société. Véritable objet-frontière, le chaudron est ainsi irremplaçable en tant que catégorie d'objet, en tant qu'« espèce».

Mais ce n'est pas tout. Chaque nganga est, pour son possesseur, un être singulier. Elle possède d'ailleurs, un nom qui lui est propre et qui la distingue des autres. Cette singularité est un paramètre voulu et activement recherché par les paleros, car elle est au fondement de la puissance de cet objet. Le pratiquant va ainsi chercher des éléments d'une morphologie singulière : une pierre en forme d'humain vaut mieux qu'une pierre informe qui n'évoque rien en particulier, un chien aux yeux particulièrement terrifiants va être préféré à un petit caniche, etc. Comme le remarque Bazin (1986 : 256) pour les boli du Mali : «Pour comprendre ce qu'est une chose-dieu, il faut [...] supposer que tous les êtres peuvent être placés sur une échelle hiérarchique en fonction de leur degré d'individuation [...] Sur ce continuum allant du particulier au singulier, du commun à l'original, du massif au ponctuel, le divin occuperait l'un des extrêmes : une chose plus singulière que toutes les autres, plus personnelle que les personnes, serait divine».

Le propriétaire d'une nganga pourra l'enrichir de nouveaux éléments (une nganga est d'ailleurs conçue de façon à ce que son volume puisse s'accroître) ; il pourra lui en substituer d'autres (les éléments peu individualisés qui n'enlèvent rien de l'identité spécifique du chaudron) mais il ne pourra jamais la remplacer intégralement. D’ailleurs, je n'ai jamais connu de palero qui ait détruit son chaudron pour en confectionner un autre. Celui qui entreprend l'opération destructive (par un rituel que l'on nomme « dar camino a la nganga », donner du chemin à la nganga) signe forcément la fin de sa carrière de palero.

Si l'irremplaçabilité du chaudron peut intuitivement être attribuée à sa singularité matérielle, il me semble qu'il faut aller plus loin. Mon hypothèse est que la matière seule, si elle n'indexe pas (au sens de Gell, 1998) une relation puissante (d'affection, de parenté, etc.), est sujette à la substitution. L'objectif est ici de montrer que l'irremplaçabilité de la 
nganga réside dans le fait qu'elle enchâsse un certain nombre de relations constitutives de l'identité rituelle de son possesseur (la relation initiatique d'une part, la relation même du palero au nfumbi d'autre part, par exemple).

Deux axes vont structurer l'argumentation : le premier est fourni par le rituel. Plus spécifiquement, je montrerai comment celui-ci, enchâssé dans le récipient les relations initiatiques, crée une personnalité pour le chaudron et établit les bases d'une véritable relation et d'une profonde affection à l'égard de l'objet. Pour cela, dans une approche microscopique et pragmatique (Bonhomme et Severi, 2009), je m'intéresserai aux interactions spécifiques ayant cours dans les rituels et aux relations qu'elles mettent en place (Barth, 1975 ; Houseman \& Severi, 2009 ; Bonhomme, 2006 ; Déléage, 2009). En considérant les rituels comme des catégories de l'action (Humphrey et Laidlaw, 1994), il deviendra ainsi possible de comprendre comment se fabrique l'irremplaçabilité dans les faits. Cette démarche, qui permet justement de mieux cerner le statut des objets, est rarement déployée dans les études afro-cubaines. Le deuxième axe sera fourni par la pratique quotidienne et visera à montrer comment le pratiquant, dans l'intimité de son temple domestique, approfondit sa relation à la nganga à tel point qu'il finit par la percevoir comme un père, un frère, l'ami le plus intime auprès duquel il cherche conseil et réconfort. Ce conseiller parvient ainsi à restructurer les arrangements domestiques et par modifier le rapport du palero à soi. Il s'agira alors de montrer comment la relation indexée par l'objet prend corps au quotidien.

\section{La relation au groupe initiatique}

Il existe deux manières différentes de conclure le pacte avec l'esprit. La première concerne les paleros expérimentés qui possèdent déjà une nganga et qui veulent s'en confectionner une autre, afin d'accroître leur efficacité magique. Dans ce cas-là, le rituel est une démarche purement individuelle : le palero se rend seul au cimetière, trouve une tombe abandonnée (non fleurie) et propose le pacte à l'esprit dont les termes sont limpides : le mort doit suivre le pratiquant dans son temple et « travailler » pour lui (accomplir des divinations, des opérations magiques, des guérisons, etc.) et le palero doit «nourrir » le nfumbi par des offrandes de sang, de rhum, de miel, de cigares, etc $^{5}$. La deuxième manière d'effectuer le pacte est réservée aux

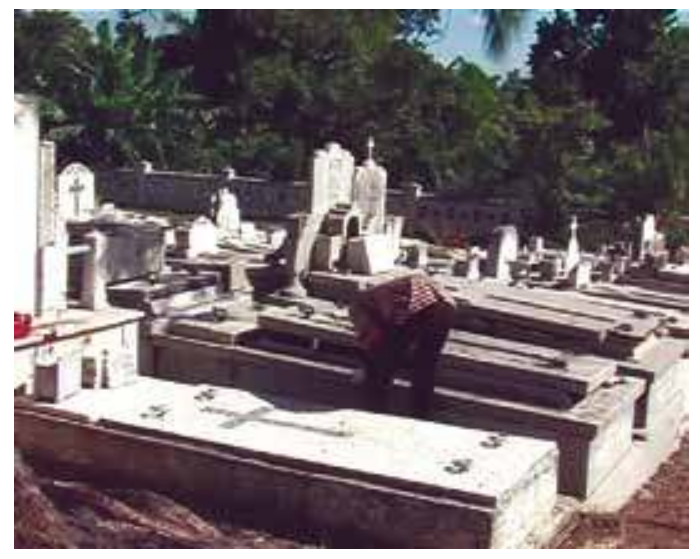
« jeunes » paleros (jeunes dans le palo) qui vont devenir possesseurs d'une nganga pour la première fois. Dans ce cas-là, le rite se déroule dans le cuarto de fundamento (temple palero) de l'initiateur. C'est ce dernier qui fournit les ossements du défunt et c'est lui qui organise le déroulement du rituel. La médiation du padrino (initiateur, littéralement « parrain ») est essentielle. Les ngangas ne naissent pas du néant mais s'inscrivent dans une lignée (rama), celle de la nganga du padrino de l'initié et sont considérées comme ses « filles ». Pour pouvoir être mises au monde, elles doivent hériter certains éléments matériels de cette dernière. Pour que puisse s'établir un transfert du «matériel héréditaire », matériel qui légitime la naissance de la nouvelle nganga, son montage doit se faire dans le sanctuaire de l'initiateur aux côtés de la «nganga-mère ». 
C'est dans cette situation que l'on doit chercher l'une des causes de l'irremplaçabilité du chaudron. En fait, on peut déduire sans équivoque que l'efficacité rituelle du possesseur d'une nganga n'est possible que si celui-ci possède un chaudron qui enferme le passé de son groupe. Le « matériel héréditaire » représente ici la parenté rituelle et plus particulièrement l'ascendance rituelle. Mais la descendance rituelle y est aussi instanciée car la nganga enferme aussi l'identité de chaque personne initiée par son possesseur. L'initiation n'est possible que si la nganga de l'initiateur a reçu le sang du novice. En fait, au cours de la cérémonie, le néophyte est incisé à différents endroits du corps. Le sang qui s'échappe de ces plaies est épongé à l'aide de morceaux de coton que l'on dépose à la surface du chaudron de l'initiateur. L'introduction de ce sang dans la nganga symbolise ainsi la relation initiatique, c'est-à-dire la relation qui unit un initiateur à son initié. Ainsi, la nganga indexe à la fois l'inscription du palero dans une lignée rituelle qui remonte loin au passé et les liens initiatiques en vigueur.

Enfin, le chaudron matérialise l'identité rituelle. Offrir son sang à la nganga est, pour les pratiquants, l'acte qui fait d'une personne un initié. Les paleros, conscients de ce fait, disent qu'ils sont les « fils du chaudron » ou encore qu'ils sont « nés sur le chaudron ».

\section{La relation au mort}

\section{La construction biographique}

Pour son possesseur, la nganga est irremplaçable surtout parce qu'elle héberge un mort de tempérament singulier. Le nfumbi va, doter le chaudron d'une personnalité humaine et d'une profondeur biographique. Cependant le mort en question est généralement un parfait inconnu. L'objectif de cette section est de mettre en lumière les procédés rituels qui viennent doter l'esprit de dispositions, d'un passé, d'une psychologie. En créant une personnalité pour le chaudron, le pratiquant définit son rapport avec lui et rend la communication plus confortable. Mais ce qui est essentiel pour notre argumentation est que cette « humanisation » du nfumbi rend possible le développement d'une relation intime entre l'esprit (et métonymiquement la nganga) et le palero.

La construction de la personnalité du mort s'inscrit dans la durée, elle est en perpétuelle évolution. Les qualités que le palero prête à son mort se modifient au gré des expériences religieuses, en fonction de ses réussites et de ses échecs. Quand un pratiquant a passé une grande partie de sa vie en compagnie d'un nfumbi, on peut être assuré qu'il s'est fait de lui une représentation riche et nuancée. Néanmoins, c'est au cours du rituel du pacte que vont être dessinés les grands traits de sa personnalité.

Puisque le palero va collaborer, voir cohabiter avec le mort jusqu'à la fin de ses jours, il va choisir ce dernier avec beaucoup de soin. Notamment, il choisira le mort qui dispose des qualités objectives, morales et psychologiques adaptées aux types de travaux auxquels il le destine. Certaines personnes ne veulent pratiquer le palo monte qu'à des fins exclusives de guérison : ils chercheront donc prioritairement un nfumbi possédant des dons curatifs, l'esprit d'un médecin par exemple. D'autres préfèrent se doter d'un nfumbi « polyvalent » disposé à accomplir tous types d'actions, qu'il s'agisse de sauver 
ou de détruire, c'est-à-dire d'un esprit sans scrupule qui ne sera pas gêné par la pitié ou l'empathie (un assassin, un politicien, un avocat...). Malgré les différences de goût en matière de nfumbis, tous s'accordent sur un point : que ce soit grâce à leur charisme, par la ruse ou encore la violence, les morts doivent avoir connu une réussite remarquable. Comme le note Hector : « si tu mets dans une nganga une personne qui n'a jamais connu la réussite, tu vas la connaître?»

Qu'il se déroule donc au cimetière ou dans le temple de l'initiateur, le rituel du pacte implique un dialogue avec le mort afin que le palero évalue ses qualités. Dans les deux cas, les méthodes divergent : au chevet de la tombe, la recomposition biographique prend la forme d'un jeu de questions/réponses, médiatisé par l'usage des chamalongos (méthode divinatoire) ${ }^{6}$. Lorsque le rite se réalise chez le parrain, la communication avec le nfumbi est, en règle générale, rendue possible grâce à la présence d'un médium. L'esprit possédera celui-ci afin de pouvoir s'exprimer oralement par sa bouche. Dans les deux cas, le dialogue avec l'esprit, banal dans sa forme et peu codifié, tourne autour des mêmes questions : il s'agit, selon les paleros, de « savoir quel est le nom du mort, où il vivait, qui il était, ce qu'il mangeait... il faut savoir tout ça pour pouvoir l'entraîner vers toi, pour pouvoir l'adapter à toi », pour pouvoir le contrôler à son profit.

Pour rendre compte du processus, je focaliserai l'analyse sur le second type de pacto, celui qui nécessite la présence d'un médium. Comme la communication avec l'esprit est, dans ce cas-là, verbale, elle offre un plus grand luxe de détails à l'observateur et permet donc de mieux préciser les modalités de la démarche de recomposition biographique. J'analyserai une séquence rituelle extraite d'un document filmique réalisé par Jean Luc Chevanne (1997). La cérémonie s'est déroulée dans le cuarto de fundamento de Pepe (un de mes propres informateurs). Certains points du rituel ont été ensuite commentés par Pepe lors d'un entretien ultérieur. Je vais focaliser l'analyse sur les moments de la construction biographique mais aussi sur ceux qui établissent les bases de l'intimité avec l'esprit.

Tous les participants sont réunis autour de la nganga unique de Pepe, la « mère » de la nganga qu'on est en train de fabriquer. Les acteurs principaux du rite sont : Pepe (65 ans), propriétaire du lieu, chef rituel et initiateur de la majorité des membres de l'assemblée ; Alberto (30 ans), l'un des filleuls de Pepe et le destinataire du chaudron dont on fait le montage ; Rosa (40 ans), qui fait office de médium.

La première demi-heure du rituel est consacrée à des salutations et à des chants rituels qui préparent Rosa à sa possession. Une fois que le mort « est venu » dans son corps, les
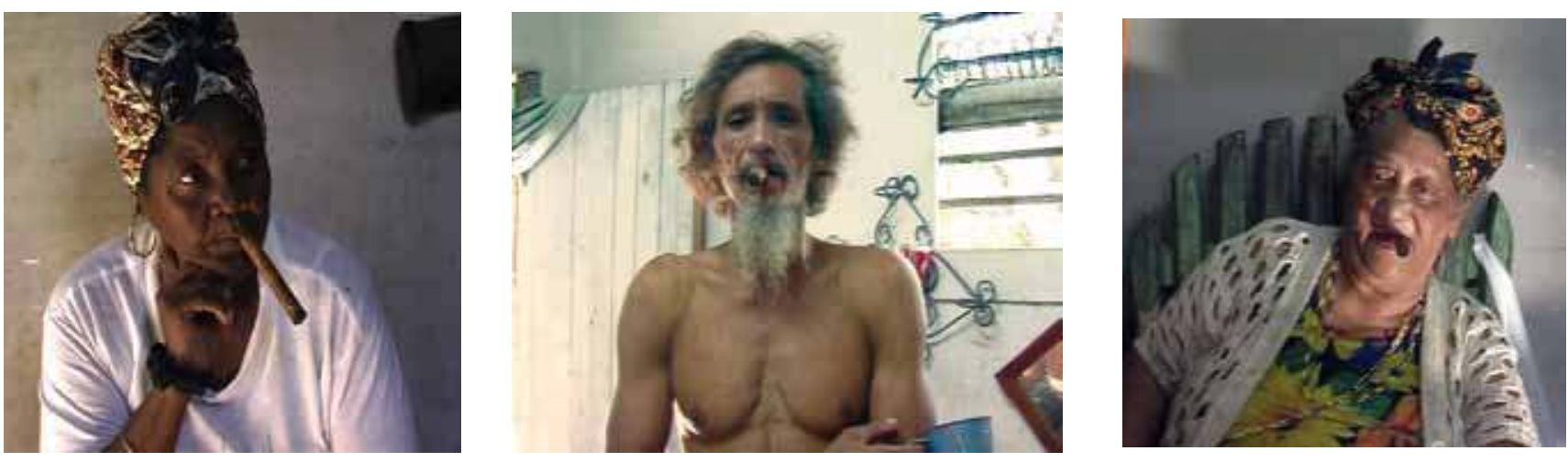


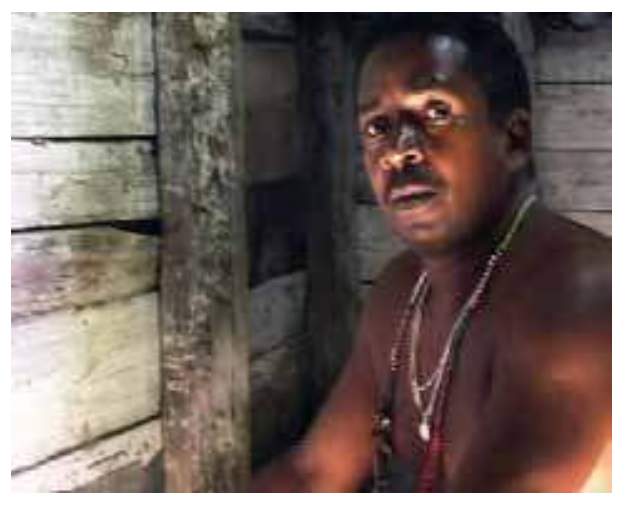

participants le saluent avec enthousiasme en lui adressant le chant de bienvenue «sala malekum, malekum sala $!^{7}$ (Pendant toute la phase de la possession, je me référerai à Rosa en utilisant le masculin comme s'il s'agissait du nfumbi). Le chant semble exciter l'esprit au plus haut point car le voilà maintenant qui s'agite avec frénésie tout en poussant des sons inarticulés.

Tout à coup, le nfumbi allume un cigare et tente de se brûler les mains avec le briquet. Pepe essaie de le calmer mais l'esprit ne prête pas attention à ses propos et tire avec frénésie de grosses bouffées de fumée de son cigare. Après quelques instants il retourne le cigare dans sa bouche, le bout incandescent à l'intérieur et continue à fumer avec avidité. De toute évidence, le nfumbi qui habite le corps de Rosa est très viril.

Celui-ci, n'ayant pas perdu de vue que ce rituel a pour vocation de créer un lien de familiarité avec son futur propriétaire, s'applique maintenant à « nettoyer » le corps d'Alberto en soufflant de la fumée sur son corps. Ensuite, il s'adonne pour un certain temps à de nouvelles démonstrations de virilité. Puis, le nfumbi semble à nouveau se prendre d'affection pour son futur palero et l'embrasse avec effusion.

Soudainement, le nfumbi salue les participants d'un geste de la main qui indique qu'il va les quitter. Mais Pepe n'est pas du même avis. Il l'invite donc à prolonger sa présence parmi l'assemblée : «Attends, petit à petit », puis il mobilise le chœur des participants pour encourager le nfumbi en chantant : « Joli, joli! ». Mais le nfumbi semble vraiment déterminé à quitter la pièce. Il salue donc une dernière fois son futur palero avant d'être pris d'une série de tremblements. Malheureusement, à ce stade de la cérémonie, aucune question personnelle n'a encore été posée à l'esprit. Alberto, qui veut en apprendre davantage sur la personnalité et sur l'histoire de son mort, entame la série de questions sans cacher son impatience :

«Qui êtes-vous?»

Le nfumbi ne répond pas. En revanche il exprime clairement son envie de vomir en mimant le réflexe émétique. Pepe tente alors de le calmer en lui faisant comprendre que les esprits ne vomissent pas (le dialogue a lieu en espagnol - la traduction est mienne - et fait intervenir des termes issus du kikongo):

«Vous n'avez rien, vous êtes un esprit. Vous êtes responsable et vous venez fort dans ce monde. Nous voulons vous connaître, savoir comment vous êtes. Vous allez devenir palero, vous allez même devenir vivant, vous aurez de la nourriture, vous aurez tout. » Et en montrant Alberto :
Pepe : «Ce compatriote naît. Awé o no awé [oui ou non] ?» Chœur : «Awé ! [oui] » Pepe : «Qu'est ce qui s'est passé, qui êtes-vous?»

Nfumbi : «Mon papa, papa Cherito [il dit en pleurnichant]. » Alberto : «Qui est Cherito?» Nfumbi : «Mon papa. »

Alberto : « Mais quel était ton nom à toi, quand tu étais une personne, quand tu étais vivant?»

Un autre palero clarifie : «Quand tu n'étais pas un nfumbi?»

Nfumbi : «Vicente. » 
Mais le nfumbi, qui probablement pense toujours à son père, se met à pleurer.

Une autre palera : «Quand êtes-vous mort?»

Alberto reformule la question : «Combien d'années ont passées depuis ta mort?»

Le nfumbi ne répond pas mais les participants insistent.

Nfumbi : «Peu de temps.»

Alberto : «Quel âge aviez-vous?»

Nfumbi : «30.»

Alberto [avec étonnement et frustration] : « 30 ? »

Nfumbi qqui comprend que cette réponse n'est pas très satisfaisante] : « 40.»

La palera : «Vous étiez Blanc ou Noir?»

Nfumbi : « Blanc. »

Pepe : « Blanc, il était blond. »

Alberto : « Mundele » [« l'homme blanc » en kikongo].

La palera : "Combien de temps a passé depuis votre mort?»

Le bakofula : «Ca fait trois, quatre ans. »

Pepe : « Non, ça ne fait pas autant de temps. »

Le bakofula : «Depuis son entrée [dans le cimetière], au moins deux ans doivent s'écouler pour le récupérer. » [Il se réfère au temps qui doit s'écouler pour transférer les ossements de la tombe à l'ossuaire.

Pepe : « Il tenait un rang très honorable [tenía posición]. Ce type était le feu, le diable. Mais c'était une très bonne personne. »

Le nfumbi fait un geste qui exprime son envie de boire.

Alberto : "C'était un ivrogne? »

Le nfumbi acquiesce de la tête.

Alberto : «As-tu voyagé ?»

Nfumbi : « Non. »

Alberto : «Tu n'as pas voyagé à l'étranger? Pense

maintenant à ce que tu as fait et où tu es allé. Laisse de côté la famille. La famille c'est autre chose. »

Pepe : «Il a des problèmes familiaux. »

Alberto: « Nous t'avons amené ici pour te garder en vie. On va [il fait un geste qui symbolise l'acte de manger] nourriture [il parle au nfumbi comme s'il parlait à un petit enfant]... nous sommes une famille, ici nous n'avons pas peur. Tu veux parler? Nous allons te donner un peu de rhum et toutes les choses que tu aimes. Nous parlons de toi, de comment tu étais quand tu étais en vie, d'où tu as voyagé. Est-ce que tu es allé au Congo ? Parce que si tu étais au Congo....Tu sais ce n'est pas tout le monde qui est allé au Congo [Le Congo est souvent confondu avec l'ancien royaume Kongo d'où le palo monte puisse ses origines. Il s'agit pour les paleros de la terre natale du culte]. »

Nfumbi : «Je suis allé en caravane. »

Pepe : «C'est possible qu'il ait voyagé quand el

Comandante [Che Guevara] y était ${ }^{8}$. »

Alberto : «Tu étais militaire?»

Le nfumbi fait un signe négatif.

Alberto : «Tu y es allé en tant que militaire?»

Nfumbi : «À Cabinda. »

Alberto : « D'accord, à Cabinda, mais en tant que militaire?»

Nfumbi : «Oui. »

Alberto : « Êtes-vous d'accord pour m'aider, moi ?

Je vais vous donner de la lumière et tout ça. »

La palera : «Dis-moi, tu as eu un infarctus après que tu es rentré?»

Les infarctus font partie des causes de mort les plus «populaires » parmi les paleros en quête de partenariat avec un mort. En effet, le caractère violent et inattendu de l'arrêt cardiaque ne laisse pas à celui qui en est victime le temps de prendre conscience de sa mort. On considère que son esprit croit donc toujours qu'il est vivant et tente désespérément de s'accrocher à la vie, ce qui le rend d'autant plus manipulable par le palero.

Nfumbi : «Oui ».

Pepe : «Viens dans ce monde, viens dans ce chaudron [d'Alberto], ce chaudron congo, dit ce tata nganga [il se réfère à lui-même] qui domine ce chaudron [son propre chaudron]. Va! Entre dans cette marmite pour aider ce fils. Pour que ce fils puisse traverser le charco azul [l'Atlantique]. Mais pour que, toi, tu puisses te sentir merveilleusement dans ce monde. »

Le nfumbi hoche la tête en signe de consentement. Il a accepté les termes de l'accord qui le lie à Alberto.

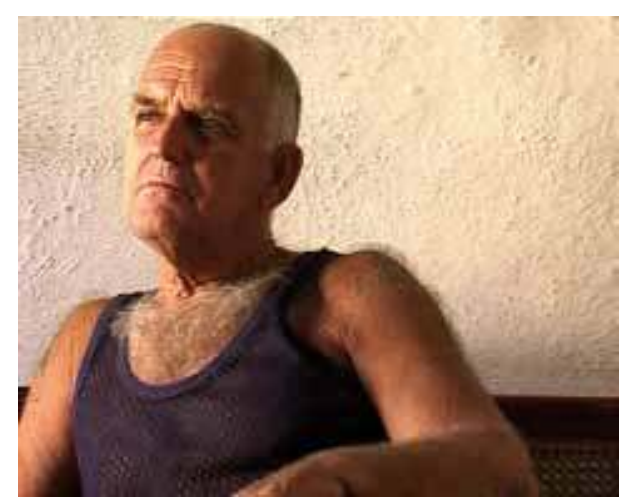



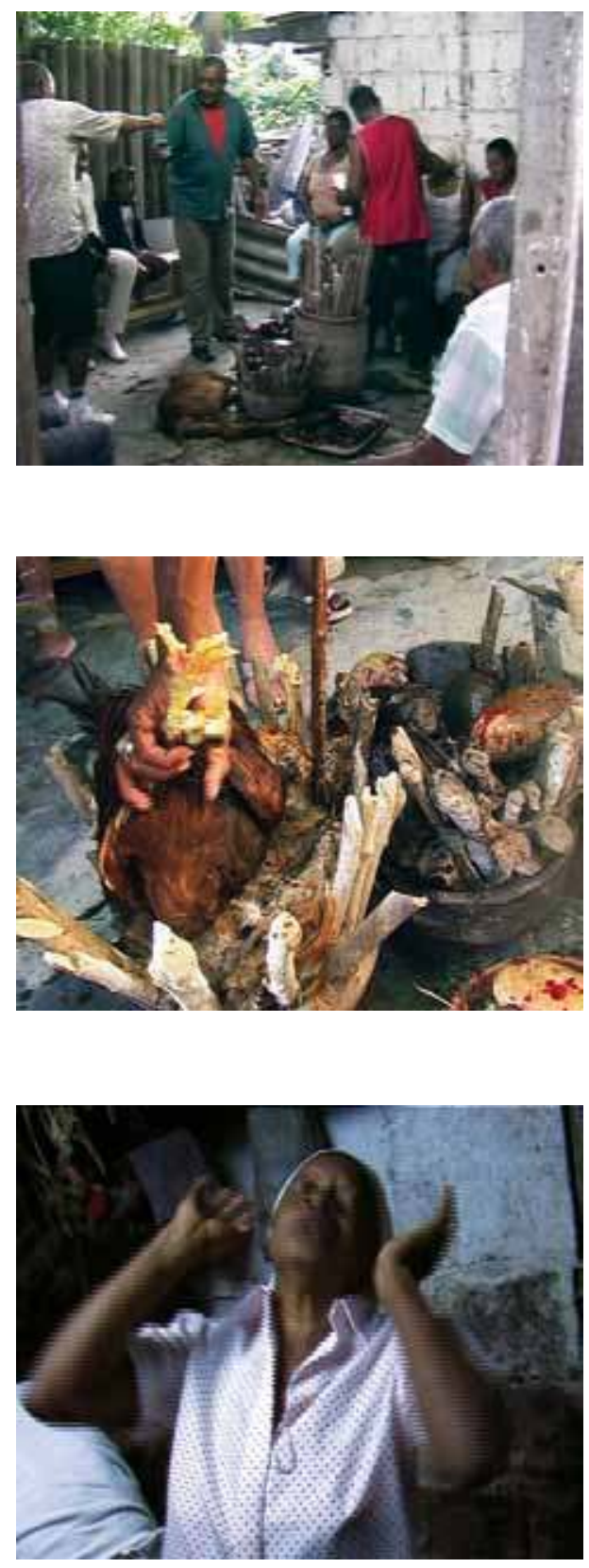

\section{Rituel, 13 décembre 2009}

II commence doucement, à cause de l'étroitesse du sanctuaire, les ngangas sont sorties dans la cour afin que tout le monde puisse assister au rituel à l'aise Esteban présente à sa ngagna. Siete Rayos: un coq avant de le sacrifier.

Ce geste vise à informer le chaudron de ce qui va se passer et lui permet de se familiariser avec l'animal quill va recevoir. La sœur d'Esteban en transe.

\section{Les ressorts de la construction biographique}

Qu'il se déroule dans un cimetière, dans la plus grande confidentialité ou dans le cuarto de fundamento, en présence d'un cercle restreint d'initiés, le rituel du pacte n'a jamais pour but de s'allier à une force impersonnelle. Il se présente toujours comme une tentative pour s'adjoindre les pouvoirs d'un nfumbi individualisé, doté d'une personnalité et d'une histoire.

Dans le cas que je viens de présenter, les rôles du médium et du public sont déterminants. Le médium, Rosa, ne peut se permettre de lancer à la légère des affirmations sur la personnalité du nfumbi, car ce dernier restera aux côtés du futur tata nganga jusqu'à la fin de ses jours. S'il possède des caractéristiques qui s'opposent aux attentes de ce dernier, leur relation risque de devenir problématique, voire conflictuelle. Le médium doit aussi prendre en considération les attentes du public. Faire reconnaître à l'assemblée des paleros les qualités du mort de la nganga, revient implicitement à légitimer la compétence magique du futur possesseur, car les pouvoirs du palero ne dépendent que de ceux de son nfumbi $i^{9}$.

L'une des techniques que le médium mobilise pour tenir au mieux son rôle consiste donc à ne répondre aux questions que de manière vague et ouverte. De cette façon, il laisse aux autres la possibilité de contribuer activement à la composition de la personnalité du nfumbi. Par exemple, lorsqu'on lui demande la date de sa mort, elle répond «peu de temps». Ce type de réponse présente des avantages : il débarrasse le médium de toute responsabilité en cas d'erreur en laissant la question en suspens et laisse l'opportunité à d'autres de fournir une réponse plausible. Donner la possibilité au public de répondre à sa place offre davantage de chances de définir pour le nfumbi une personnalité acceptable selon les critères de la communauté. ${ }^{10}$

Mais de tous les participants, c'est l'avis du futur tata qui est le plus essentiel, car après tout, c'est de son propre nfumbi qu'il s'agit et il importe que son histoire lui plaise. Il ne faut donc pas s'étonner quand Rosa revient sur certaines de ses déclarations quand elles ne provoquent pas l'enthousiasme d'Alberto. Même si c'est parfois au prix de sa crédibilité. Quand elle comprend par exemple qu'un nfumbi de 30 ans est bien trop jeune pour Alberto, elle le vieillit instantanément de 10 ans. Plus tard, le médium admet que le mort a voyagé au Congo pour combler les désirs d'Alberto (expliquer : ex. il s'agit là d'une puissante référence à l'ancestralité du culte, ou n'importe quoi d'autre) - alors qu'il a déclaré quelques minutes avant que celui-ci n'avait jamais quitté le pays. Pour maintenir la crédibilité du médium, qui menace de s'effondrer à tout moment, Pepe intervient pour appuyer le scénario de son médium : «Le nfumbi a probablement voyagé au Congo pendant la période où Che Guevara y était. »Comme nous pouvons le constater, la participation d'Alberto à la définition de la personnalité du nfumbi est donc écrasante. Il oriente les réponses du mort jusqu'à obtenir le profil adéquat. 


\section{Opération magique : préparation d'une nsunsundamba}

Les photos ci-dessous ont été prises chez un palero lors de la préparation d'un artefact rituel : la nsunsundamba. Cette chouette est en effet nécessaire à la guérison du client.

Comme tout artefact palero, la nsunsundamba est arrangée afin de servir de réceptacle à de nombreuses puissances de la nature. Avant de préparer son artefact rituel, le palero, s'est purifié le corps avec ce cigare. Le feu qui le brûle est censé brûler toutes les mauvaises influences capables d'entraver le bon déroulement de la fabrication.

Le prêtre a déjà vidé et asséché la chouette. Il est maintenant en train de la remplir de substances magiques, similaires à celles qu'on introduit d'habitude dans une nganga (branches rendues en poudre, herbes, pierres, parcelle d'os humain, etc.).

Le palero, incise sa langue : son sang précieux, va donner de la vie à la nsunsundamba et il va accroître son efficacité. La nsunsundamba est prête et visiblement contente de l'attention qu'on lui a porté. Ayant désormais une place dans le sanctuaire, elle peut commencer son « travail ».
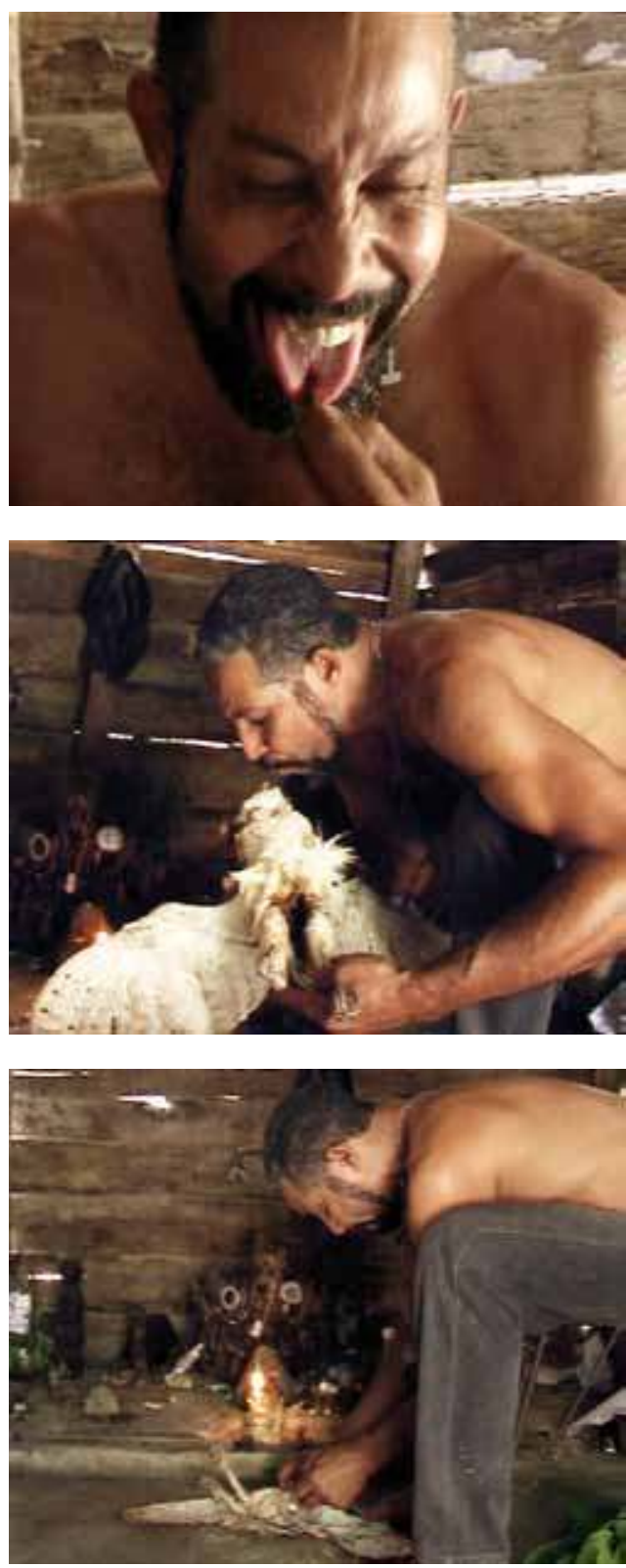
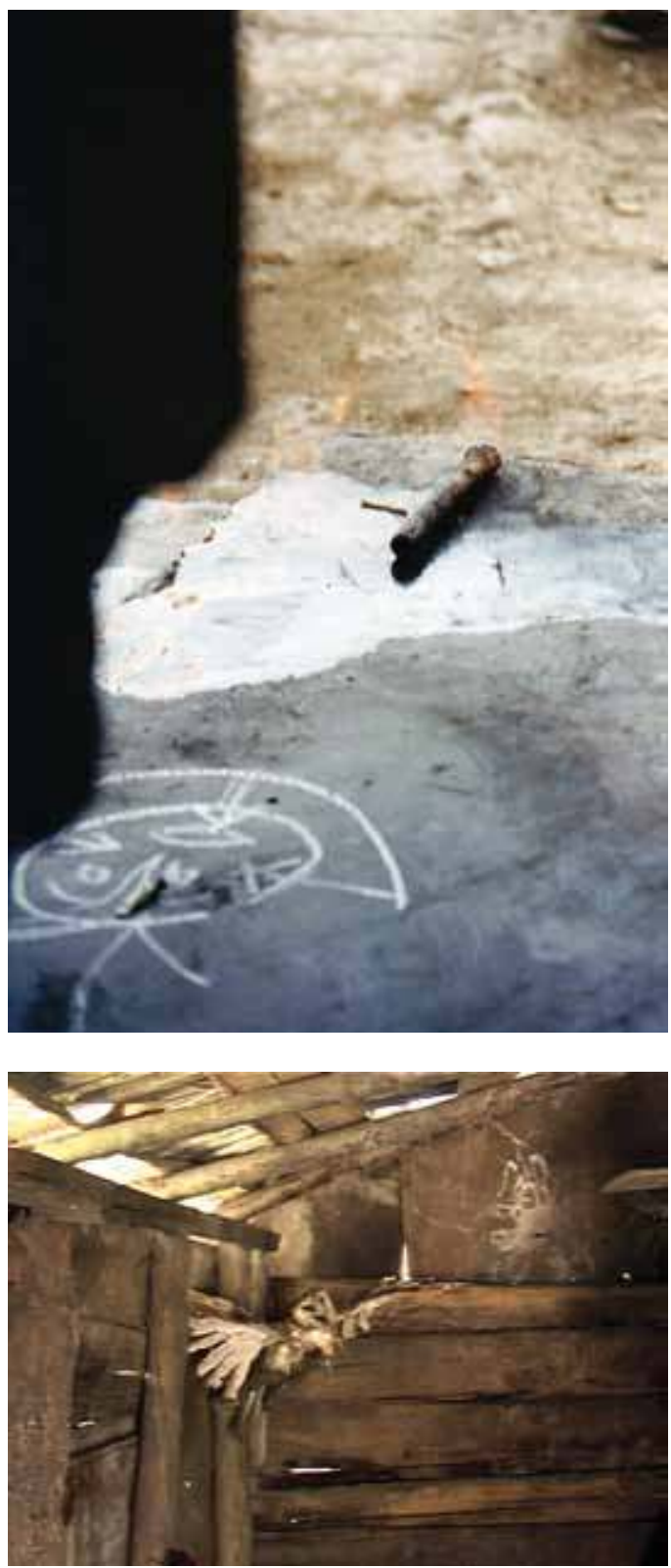
À la fin de cette séquence, le nfumbi d'Alberto est un homme blanc de 40 ans. Comme l'a précisé Pepe, il était de « rang honorable » avec un tempérament explosif (« ce type était le feu, le diable ») mais avec un bon fond (« c'était une très bonne personne »). Même si chaque palero attribue des caractéristiques différentes à son mort, les images de réussite et de force sont sans surprise très souvent revendiquées pour leur nfumbi.

Mais cette séquence rituelle ne fait pas que dresser un portait heureux du mort. Elle pointe aussi certains de ses vices et de ses travers : on y apprend notamment que le mort d'Alberto a été un ivrogne et qu'il a connu de graves problèmes familiaux. Souvent les nfumbis possèdent ces deux caractéristiques. D'une part, la dépendance à l'alcool accroît la dépendance au palero, le nfumbi obéissant au pratiquant afin d'obtenir sa « dose ». D'autre part, quand un esprit a été privé d'amour familial, il est davantage susceptible d'exprimer sa reconnaissance et sa fidélité à celui qui l'aimera. ${ }^{11}$

Nous le voyons clairement, le « jeu » rituel est doux avec Alberto : les protagonistes du rite accomplissent modestement leur rôle sans trop s'adonner à des démonstrations d'autorité afin que celui-ci puisse construire un « dieu » à sa mesure. La labilité du dialogue identificatoire permet au futur propriétaire du chaudron de s'allier à un mort puissant, capable de rivaliser avec les nfumbis des paleros les plus réputés. Mais si la force du mort est une condition pour qu'Alberto puisse développer une confiance dans ses compétences religieuses, elle ne doit pas pour autant l'intimider. Ainsi, dans un mouvement opposé, le dispositif rituel, en mettant l'accent sur « l'humanité » de l'esprit, crée les bases d'une complicité avec le mort.

Certains pratiquants d'ailleurs, en faisant de la complicité la composante essentielle de leur relation à l'esprit, finissent, à travers les interminables conversations avec le chaudron, par construire sa personnalité à leur image. Puisque c'est le palero qui détermine le contenu des dialogues par un jeu de projections et de transferts, on ne peut s'interdire de penser que c'est une certaine version de lui qu'il recompose chez son mort.

Que le mort acquière une personnalité semblable à celle de son palero ou qu'il dispose de traits caractériels qui favorisent le développement d'une amitié (même âge que le palero, amour pour le rhum, traumatismes familiaux qui le rendent « sensible »), dans tous les cas il s'agit pour le palero d'un humain à qui on peut parler d'égal à égal, « d'une personne comme les autres ».

\section{L'affection pour le mort}

L'amour pour le nfumbi est la conséquence logique de cette situation. D’ailleurs, dès le rituel du pacte, le mort a dirigé toute son affection vers Alberto et a ainsi produit les premiers signes d'une relation émotionnelle et exclusive. Je rappelle que les termes du pacte correspondent théoriquement à un échange réciproque de services matériels. Or, lorsqu'on a assisté à des rituels paleros on en vient à se former l'image d'un être doté d'une sensibilité ordinaire, parfois enfantine, dont les traits de caractère contrastent de manière éclatante avec l'image cruelle de l'assassin, du psychopathe, de l'alcoolique que l'opinion commune attribue d'habitude à ces esprits. Comme nous venons de le voir, les nfumbis pleurent comme des enfants pour leur père, ils manifestent de l'affection pour leur palero (nous avons vu que le nfumbi prend Alberto dans ses bras affectueusement), ainsi que leur envie de le protéger (le médium se précipite pour «nettoyer » son futur tata nganga). En bref, lors de leurs apparitions, les nfumbis se montrent particulièrement sensibles, étrangement doux et tendres. 
Évidemment, c'est le rite même qui assigne au mort ce tempérament. L'accueil qu'on fait au nouveau nfumbi, l'ambiance dans laquelle naît la nouvelle nganga est fort émotive et particulièrement affectueuse. Tout au long du rite, les initiés chantent « ma bonne nganga! », « mon beau palo [nganga] », « joli, joli ! ». Pepe encourage le nfumbi à poursuivre le rite malgré sa fatigue en prenant son médium dans ses bras, en incitant l'assemblée à lui chanter «nganga avec le cœur! ». Cette expression quasi-obligatoire d'affection pour le nfumbi ne peut le laisser impassible; il doit s'adapter au schème interactif établi par le rituel. Dans notre cas, c'est d'ailleurs après ce paroxysme affectueux que l'esprit éclate en larmes et réclame son cher papa. Avec les effusions d'amour fraternel dont le nfumbi a gratifié Alberto, le ton est donné pour que le mort-chaudron agisse dans la vie du palero à un niveau personnel.

Le montage matériel du chaudron qui suit la séquence rituelle que l'on vient de décrire, implique un acte rituel qui creuse davantage le lien exceptionnel qui unit le palero à son nfumbi. Notamment, une fois que tous les éléments sont installés dans le chaudron, on doit leur infuser de la vie. Car même si son ingrédient principal est un mort, la nganga est un être vivant qui partage certaines caractéristiques propres à la vie (faim, soif, nervosité, humidité, chaleur). Or, la nganga ne peut être animée qu'à condition d'avoir reçu le sang de son futur propriétaire. Le palero « [...] pratique une incision dans son bras et laisse couler quelques gouttes de sang dans le chaudron » (Cabrera 2003 [1954] : 140). Le sang du propriétaire agit à la manière d'une transfusion, il donne vie à ce qui n'était encore qu'un assemblage de forces éparses sans ordre et sans vie. Le sang renferme pour les paleros une force vitale capable de recomposer la matière morte. Ce geste constitue une marque extrême de fraternisation, un pacte de sang. Mais sa dimension irréversible ne doit pas être perçue qu'à un niveau symbolique. Le nfumbi connaissant désormais l'odeur du sang du palero, celui-ci n’aura plus aucune échappatoire et devra toujours garder le mort à ses côtés. Il s'agit bien là d'une rhétorique mais sa conséquence est que le palero croit que le mort « le suit comme une ombre », qu'il « vit dans son corps», ou encore qu'il est « toujours à côté de lui, pour lui chuchoter des choses à l'oreille». Cette proximité spatiale et ontologique conduit le pratiquant à penser qu'il prend toutes ses décisions, ou presque, en concertation avec son mort.

\section{La vie avec les morts}

Une fois la nganga montée, le palero l'emmène chez lui. S'il dispose de l'espace nécessaire, le mort devient le locataire permanent d'une pièce située au fond de son jardin, le cuarto de fundamento, le temple palero, spécifiquement construit pour héberger le chaudron. La pièce est exclusivement réservée à la pratique du palo monte : aucun non-initié, pas même la famille de son propriétaire ne peut y pénétrer sans son autorisation. Ce lieu, soigneusement séparé du reste de l'habitat du pratiquant, de sa famille, du monde séculier, favorise une pratique solitaire au sein de laquelle la relation intime avec l'esprit peut s'approfondir ${ }^{12}$

Le palero visite son chaudron quotidiennement ou presque. La nganga est pour les paleros un être « vivant » qui a besoin d'une attention régulière. Plus précisément, le pratiquant doit chaque jour «nourrir » le chaudron avec du rhum, de la fumée de 

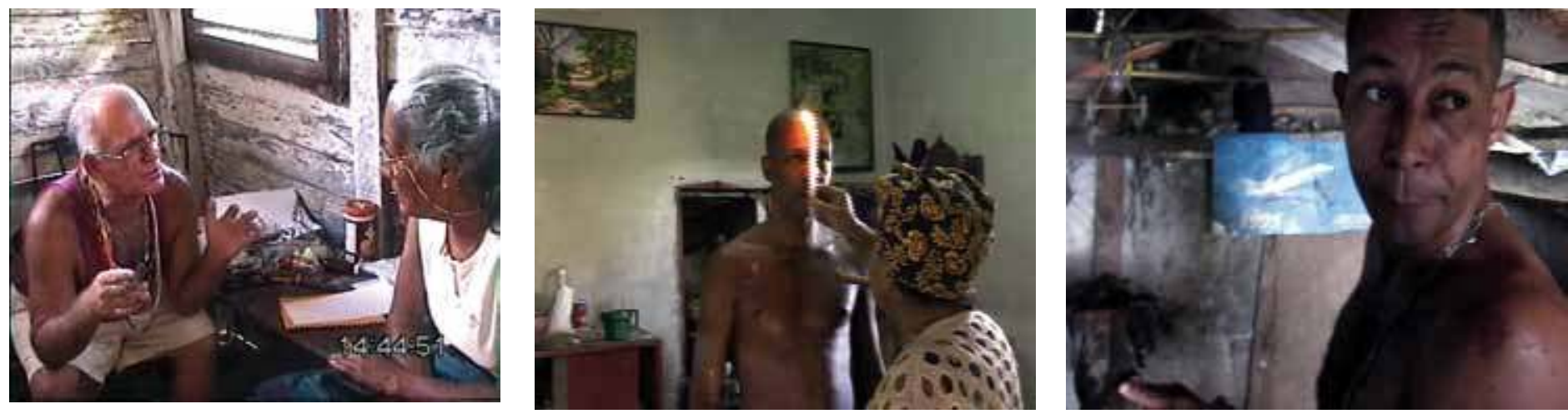

\section{Le palo monte et les problèmes de la vie quotidienne}

Nombreux sont les Cubains à faire appel aux services d'un palero afin de résoudre magiquement un problème personnel.

- Photo 1, on voit le palero Jorge qui consulte une cliente.

- Photo 2, on voit la palera Marelis « nettoyer » rituellement un de ses initiés qui souffre de problèmes de santé. En fait, problèmes de santé, maux conjugaux, familiaux, professionnels figurent systématiquement dans l'étiologie de l'adhésion au palo monte. Dans un premier temps, la personne affligée fait appel aux services du spécialiste rituel et tente d'éliminer les causes de sa souffrance par une simple consultation. Le prêtre propose généralement un traitement magique. Si l'infortune persiste, celui-ci peut prescrire au patient l'initiation afin de lui assurer un bien-être durable.

- Photo 3, on voit Pedro Tomás dans son temple domestique. La maquette de l'avion en plastique suspendue au toit et l'affiche jaunie figurant un Boeing au décollage ne constituent pas des décorations. Leur fonction est d'aider une personne à quitter le pays, en général pour émigrer. Cela fait partie des demandes les plus courantes auxquelles sont confrontées les paleros. Et ce n'est pas un hasard si ces objets figurent de manière saillante dans son sanctuaire. Le grand rêve de ce pratiquant est de s'exiler à l'étranger. Et chaque jour, il sollicite sa nganga pour qu'elle lui permette de réaliser son projet.

cigare et du miel. Le fait que celui-ci ait des besoins humains ne contredit pas pour les pratiquants le caractère « divin » de la nganga. Cet artefact constitue l'unique source de savoir et, pour les paleros, les visites quotidiennes dans le cuarto de fundamento sont l'occasion de demander toutes sortes de conseils. Par différents procédés divinatoires, par la possession rituelle ou encore par un dialogue télépathique, l'objet communique au pratiquant la meilleure manière de procéder pour parvenir à ses fins, pour obtenir certaines connaissances, pour aider un tiers ou bien pour lui nuire. Parfois, le chaudron suggère à l'officiant des techniques, des procédés, des filtres magiques inédits qui sont autant de nouvelles pratiques qui viendront enrichir l'appareil rituel de la « familia religiosa »(la famille religieuse, le groupe initiatique).

Mais le palero n'interroge pas exclusivement le chaudron pour enrichir sa pratique religieuse et magique. Ce qui l'intéresse avant tout est de savoir comment améliorer sa condition personnelle, matérielle et relationnelle. L'accès immédiat à un être réputé omniscient rend le palero dépendant de son avis en ce qui concerne les démarches à faire dans sa vie professionnelle mais aussi pour révéler les intentions cachées de ses proches ou déjouer les actes malveillants de ses voisins. De toute manière, le mort devient très rapidement l'interlocuteur privilégié du palero :

« Avant de faire quoi que ce soit, j'interroge toujours le mort. Est-ce que je peux aller au travail aujourd'hui ? Est-ce que je peux coucher avec cette femme? En fonction de ce qu'il va me dire, j'agis ». (Esteban)

La vie devient ainsi plus facile : le mort lève les doutes et les suspicions, les relations avec les autres peuvent être vécues avec une plus grande quiétude et un plus grand détachement. Mais en dehors du réconfort psychologique apporté par le dévoilement des états d'âme d'autrui, l'accès immédiat à un être réputé omniscient qui peut déjouer les actes malveillants de ses voisins, qui peut révéler les intentions cachées de ses proches ou encore guider le palero dans les complexités des rapports conjugaux, n'est pas sans conséquences pratiques. L'introduction d'une nganga dans une maisonnée modifie 
les rapports du palero avec son entourage en agissant d'une certaine manière sur sa psychologie : modification du registre de son attention (faire, par exemple, davantage attention aux détails de son environnement), de son rapport à la décision (en interrogeant les chaudron sur la pertinence d'une action, il dissipe ses doutes et facilite ainsi la prise de décisions), du rapport à soi (le chaudron devient progressivement une extension/version de soi, une « altérité constituante », pour reprendre la formulation de Philippe Erikson (1986). La nganga vient reconfigurer les arrangements domestiques : être intentionnel et influent, le chaudron du palero s'immisce dans la relation avec la conjointe, devient le guérisseur de ses enfants, un juge des situations, un nouveau membre de la maisonnée à qui l'on doit consacrer du temps et de l'argent.

Au regard des bénéfices apportés par la consultation de la nganga et compte tenu de la très grande facilité avec laquelle les informations sont obtenues (il suffit de poser sa question au mort puis d'enregistrer sa réponse par la voie des chamalongos, il n'y a pas de sacrifice à accomplir, la réponse est généralement immédiate, etc. $\left.{ }^{13}\right)$, la fréquentation du chaudron devient rapidement une habitude voire une dépendance.

De cette fréquentation assidue motivée au départ par l'intérêt émerge généralement une relation bien plus complexe où dominent des sentiments puissants et désintéressés:

« Avec le temps, il va gagner peu à peu ta confiance, oui. Pour moi, c'est mon frère, mon ami, ma confiance. Ma nganga pour moi, c'est ma confiance. » (Hector)

« Pour moi la nganga est mon père... Et depuis que j'ai perdu mon papa en 1983, elle est devenue même bien plus pour moi. La nganga est quelque chose de très grand qui m’accompagnera jusqu'aux derniers moments ». (Pedro Tomás)

\section{$\&$}

Dans cet article nous avons essayé de voir pourquoi chaque nganga est pour son possesseur un être irremplaçable. Tout d'abord, nous avons vu que l'irremplaçabilité de l'objet se présente comme le produit d'une réalité relationnelle qui se donne à voir à travers la matérialité de l'objet. Le chaudron symbolise l'appartenance à une lignée rituelle, la relation entre initié et initiateur et tous les liens initiatiques en vigueur. Se débarrasser de son chaudron ou l'échanger contre un autre signifierait donc le désengagement des liens de la parenté rituelle. Les implications de cette situation seraient majeures: l'initiation dans le palo monte introduit une personne dans un groupe; le montage de la nganga, effectué d'habitude plusieurs années après la première initiation, indexe généralement la profonde implication de l'individu dans ce groupe. Jeter ou remplacer son chaudron, fruit d'une collaboration (voire amitié) de longue haleine, entraînerait la sortie de la personne du groupe initiatique. Et pour celui qui a fondé son propre groupe, se débarrasser de sa nganga reviendrait à « désadopter» ses « enfants » rituels. D'autant plus qu'à Cuba, parenté rituelle et réseaux de voisinage et d'amitié sont souvent inextricablement liés de sorte que celui qui se désengage de ses liens initiatiques risque de bouleverser l'ordre de sa vie sociale. 
Mais le rituel pose les bases de l'irremplaçabilité de l'objet à plusieurs niveaux. D'un point de vue fonctionnel, en singularisant son profil et son histoire personnelle, il fait déjà de sa substitution, une opération délicate : il serait difficile de trouver un mort avec les mêmes dispositions. D'un point de vue psychologique et émotionnel, le rite creuse davantage cette difficulté en colorant la relation qui unit le palero à sa nganga de familiarité, d'affection et d'intimité. Et, plus important encore, il induit chez le palero l'idée que son parcours dans le culte est inextricablement lié à la relation qu'il a nouée avec son chaudron.

En sortant du cadre rituel, nous avons aussi considéré le rôle de la nganga dans la vie quotidienne du palero et dans l'instauration d'un certain type de rapport à lui-même et à sa famille. Remplacer cet objet, c'est donc mettre en question ses décisions précédentes, son équilibre familial, son identité.

Définitivement, l'irremplaçabilité de la nganga tient au fait de sa très grande implication dans la vie tant religieuse que séculière du pratiquant.

\section{NOTES}

toutes les illustration sont extraites de scènes vidéos faites par l'auteur

1. Pour les travaux les plus anciens sur la santeria, cf. Ortiz (2001 [1906]) ; Cabrera (2003[1954], 1974) ; Lachatañeré (2001 [1938 et 1942]). Pour les plus récents santeria, cf. Brown (2003a);Konen (2009);Holbraad (2005, 2007, 2008), Gobin (2008, 2009). Pour les recherches sur la société secrète abakuá, cf. Brown (2003b) et Morel-Baro (2010).

2. Et à l'exception des remarquables travaux ethnographiques de Lydia Cabrera (2003[1954], 1979), les études sur le palo monte sont plutôt d'inclinaisons cosmologiques (Bolívar Arostégui et González Díaz de Villegas 1998), philosophiques (Figarola 2001, 2006a, 2006b ; Ramón Ochoa 2007, 2010a, 2010b), ou linguistiques (Fuentes Guerra \& Armin Schwegler 2005).

3. Pour une analyse minutieuse du processus de la fabrication d'une nganga, voir Kerestetzi (2011).

4. Toutes les conditions événementielles de la découverte des matériaux, lorsqu'elles sont communiquées, enrichissent aussi la biographie de la nganga et renforcent ainsi sa réputation (Kerestetzi, 2011).

5. La profanation d'une tombe est punie à Cuba de 30 ans d'emprisonnement. Ainsi, certains, pour éviter le risque, préfèrent s'arranger avec les gardiens des cimetières pour se procurer des ossements une fois qu'ils ont été transférés à l'ossuaire.

6. Il s'agit de quatre morceaux d'écorce de coco coupés en rond, dont l'un côté est convexe et l'autre concave. Le palero pose sa question est puis jette les ces rondelles divinatoires. La combinaison du nombre des côtés convexes et des côtés concaves donne une configuration spécifique qu'on appelle letra (lettre) : pour le palero il s'agit de la réponse du mort.
7. Cette salutation arabe est une salutation rituelle usuelle dans le palo monte et doit probablement son origine soit aux marchands arabes présents à Cuba soit aux esclaves Mandingues, Wolof, Fula, etc. qui ont été islamisés avant leur arrivée à Cuba (Ortiz, 2001[1906]).

8. En 1965 Che Guevara est allé avec un contingent de Cubains au Congo avec le rêve d'étendre la Révolution en Afrique.

9. Pour que le médium ne commette pas d'impairs, il est donc préférable qu'il ait une longue expérience de ce type de cérémonies et qu'il soit au fait de la tradition du groupe initiatique dans lequel il officie. Car les préférences des communautés religieuses en matière de nfumbis sont éminemment variables. Elles dépendent aussi bien de la tradition dans laquelle elles s'inscrivent que de la personnalité du chef en activité. Enfin, il est bon que le médium soit un proche du futur tata nganga (possesseur de nganga) afin de définir une personnalité du nfumbi au plus proche de ses attentes. Autant le dire tout de suite, Rosa est loin de remplir tous ces critères. À l'époque où a lieu le rituel, elle est loin d'être une palera assidue. De temps à autre, seulement, elle fait office de médium pour les cérémonies de Pepe. D’Alberto, elle ne connaît rien.

10. Si Rosa avait dit que le nfumbi était décédé il y a 20 ans, elle aurait probablement frustré Alberto et mis l'assemblée dans l'embarras. Mais dans notre cas, la réponse évasive a automatiquement été complétée par des données conformes aux attentes du groupe (2-3 ans). La durée d'existence post mortem optimale est infiniment variable selon les groupes considérés et certains préfèrent que les morts aient acquis une plus grande expérience du monde 
des esprits avant de les piéger dans leur nganga. Mais la majorité d'entre eux, comme cela semble être le cas ici, précisent qu'il ne faut tout de même pas exagérer l'ancienneté du mort ; il semble tout aussi important que celui-ci se souvienne de sa vie antérieure, qu'il n'ait pas perdu son « humanité » (terme employé par Figarola, 2006 : 38).

11. Chacune des caractéristiques attribuées à l'esprit aura ensuite un impact sur la pratique religieuse de son palero, sur le style de ses rituels. Considérons, par exemple, la dépendance à l'alcool. Quand les nfumbis sont des alcooliques, pendant les rites, ils demandent aux participants de consommer de grandes quantités d'alcool. Tous les nfumbis boivent et le rhum est présent dans chaque activité palera. Or, c'est la quantité qui fait toute la différence. Dans certaines maisons de culte, où le nfumbi n'est pas un alcoolique la consommation d'alcool est modérée. Les participants pourront boire quelques gorgées mais le chef rituel restreindra l'usage de l'alcool afin d'éviter les cas d'ivresse. La simple relation du nfumbi à l'alcool peut ainsi avoir des conséquences importantes sur l'ambiance qui règne dans un rituel : euphorique, festive, solennelle, sévère, ennuyeuse parfois.

12. Si par la suite le pratiquant se confectionne de nouveaux chaudrons, il les installera dans ce même temple, aux côtés de sa nganga initiale qui est en règle générale son interlocuteur principal, el jefe (le chef).

13. Il faut noter ici que la concurrence et la méfiance est une caractéristique des relations entre paleros de sorte quau sein même de la relation initiatique le secret structure la transmission des connaissances. Aussi, en dehors des cas particuliers où l'initié est un ami proche ou un parent de son instructeur, c'est-à-dire une personne de confiance, celui qui veut obtenir un savoir de ses initiateur éprouve un sentiment de frustration.

\section{RÉFÉRENCES}

Barth, F. 1975 Ritual and knowledge among the Baktaman of New Guinea, New Haven : Yale University Press.

Bazin, J. 1986 « Retour aux choses-dieux », Le temps de la réflexion, 7 : 253-273.

Bonhomme J. \& Severi C.(dir.)2009 Parole en actes, Paris, L'Herne (Cahiers d'anthropologie sociale, 5).

Brown David H. 2003a Santeria Enthroned : art, ritual, and innovation in an Afro-Cuban religion, Chicago ; London: The University of Chicago Press.

- 2003b The Light Inside : Abakuá Society, Arts and Cuban Cultural History, Washington ; London : Smithsonian Books.

Cabrera L. 2003 |1954] La Forêt et les Dieux (El Monte), Paris : Jean Michel Place.

- 1974 Yemaya y Ochún, Madrid : S.N.

Deleage P. 2009 "Les savoirs et leurs modes de transmission dans le chamanisme sharanahua », Cahiers d'anthropologie sociale/ Paroles en actes, $5: 63-85$.

Erikson P. 1986 Altérité, tatouage, et anthropophagie chez les Pano : la belliqueuse quête du soi , Journal de la Société des Américanistes de Paris, LXXII : 185-210.

Figarola J. J. 2001 Para un nuevo acercamiento a la nganga, Del Caribe, 35 : 23-31.

- 2006a La Brujería Cubana : El Palo monte, Aproximación al Pensamiento Abstracto de la Cubania, Santiago de Cuba : Editorial Oriente.

- 2006b Cuba, la gran nganga (algunas prácticas de la brujería). Santiago de Cuba : Ediciones Caserón.

Fuentes Guerra J. \& Schwegler A. 2005 Lengua y rito del Palo monte Mayombe : dioses cubanos y sus fuentes africanos. Madrid : Ediciones Iberoamericana

Gell A.1998 Art and agency: an anthropological theory. New York ; Oxford : Oxford University Press.

Gobin E. 2008 La Iniciacion de extranjeros en la santeria y el culto de Ifa cubanos : transnacionalizacion religiosa, conflictos y luchas de poder en La Habana. In K. Argyriadis, R. de la Torre, C. Gutierrez \& Al. Raíces en movimiento. Prácticas religiosas tradicionales en contextos translocales. Zapopan; Jalisco : IRD/CEMCA/CIESAS.

— 2009 À propos des cultes d'origine yoruba dans la Cuba socialiste (1959 à nos jours), Cahiers des Amériques Latines, 57-58: 143-158. 
Holbraad M. 2005 Expending multiplicity : money in Cuban Ifá cults, Journal of the Royal Anthropological Institute, 11 (2) : 231-254.

- 2007 The power of powder : multiplicity and motion in the divinatory cosmology of Cuban Ifá (or mana, again). In A.Henare, M. Holbraad \& S. Wastell (dir.), Thinking Through Things : Theorising Artefacts Ethnographically. London; New York: Routledge.

— 2008 Definitive evidence, from Cuban gods, The Objects of Evidence, Journal of the Royal Anthropological Institute: 93-109.

Houseman M. \& Severi C. 1994 Naven ou le donner à voir. Essai d'interprétation de l'action rituelle. Paris : École des Hautes études en Sciences Sociales.

Humphrey C. \& Laidlaw J. 1994 TheArchetypal actions of ritual : a theory of ritual illustrated by the Jain rite of worship. Oxford: Clarendon Press.

Kerestetzi K. 2011 Fabriquer une nganga, engendrer un dieu (Cuba). Images re-vues/Figurer les invisibles, 8.

Konen A. 2009 Rites divinatoires et initiatiques à La Havane : la main des dieux. Paris : L'Harmattan.

Lachatanere R. 2001 [1938 et 1942] El sistema religioso de los afrocubanos. La Havane : Editorial de Ciencias Sociales.

Macgaffey W. 1986 Religion and society in Central Africa : the BaKongo of Lower Zaire. Chicago ; London : The University of Chicago Press.

— 1993 The Eyes of understanding : kongo minkisi. In M. D. Harris (dir.) Astonishment and power : Kongo minkisi \& The art of Renée Stout. Washington : The national museum of African art by the Smithsonian Institution Press.

Morel-Baro G. 2010 Enjeux de pouvoir, pouvoir en jeu et institutionnalisation de la société secrète

abakuá à La Havane, EchoGéo, 12.

Ochoa Todd R. 2007 Versions of the Dead : Kalunga, Cuban-Kongo Materiality, and Ethnography, Cultural Anthropology, 22 (4) : 473-500.

- 2010a Prendas-Ngangas-Enquisos : turbulence and the influence of the dead in Cuban-Kongo material culture, Cultural Anthropology, 25 (3) : 387-420.

- 2010b Society of the Dead: Quita Manaquita and Palo praise in Cuba. Berkeley; Los Angeles; London : University of California Press.

Ortiz F. 2001 [1906] Los negros brujos : apuntes para un estudio de etnología criminal. La Havane : Editorial de Ciencias Sociales.

Young J. R.2007 Rituals of resistance : African atlantic religion in kongo and the lowcountry south in the era of slavery. Louisiana State University Press.

Wirtz K. 2007 Ritual, Discourse, and Community in Cuban Santeria : Speaking a Sacred World. Gainesville : University Press of Florida

- Filmographie

Chevanne, J.-L. 1998 Fondement d'une nganga de Siete Rayos. 87'. 


\section{RÉSUMÉ}

Un mort pour son chaudron. Ou comment faire du dieu-objet du palo monte un être irremplaçable (Cuba). La nganga, chaudron de fer qui condense la présence d'un mort, est l'objet focal de la pratique du culte afro-cubain du palo monte. Source unique de pouvoir et de savoir pour les adeptes (paleros), il n'est pas un aspect du culte qui ne requiert sa présence ou sa médiation (rites, opérations magiques, consultations). Élément structurant de la pratique religieuse, la nganga se présente aussi comme un objet-frontière : marque de fabrique des paleros, elle est le symbole du culte et de ses prêtres pour le reste de la société. Ces raisons font que le chaudron des paleros est irremplaçable en tant que catégorie d'objet. Mais chacune des ngangas est pour son possesseur un être singulier et irremplaçable. L'objectif de cet article est de mettre en lumière les processus suivant lesquels un adepte ne peut imaginer sa vie sans son chaudron. La thèse défendue ici est que l'irremplaçabilité de la nganga tient à sa capacité d'indexer un certain type de relations. Plus spécifiquement, il sera question de monter comment à travers certains processus rituels, la nganga devient l'objectification d'un ensemble de relations constitutives de l'identité rituelle de son possesseur (comme par exemple la relation initiatique). Ensuite, en focalisant sur la vie quotidienne, nous verrons que la nganga finit par restructurer les arrangements domestiques et par modifier le rapport de l'adepte à soi. Il sera donc question de montrer que remplacer ou détruire sa nganga signifierait pour le palero une remise en question radicale de son existence.

\section{ABSTRACT}

A dead for a caldron. Or how the god-object from palo monte becomes an irremplaceable entity (Cuba). The nganga, an iron cauldron which embodies a dead man's spirit, is the central object of the Afro-Cuban religion of palo monte and the unique source of the worshippers' (paleros) power. All aspects of this religious practice rituals, magical operations, consultations, divinations, etc. - require indeed its mediation. The nganga is a structural element of this religious practice and also a boundary object: this cauldron is palo monte's symbol, the paleros' trademark. Therefore, the nganga is irreplaceable as a category of object. However, for their owners, each nganga is unique and irreplaceable. This article highlights the processes which prevent worshippers from imagining their lives without their cauldron. The core argument I wish to defend here is that the nganga's irremplaceability is due to its capacity to index certain kinds of relationships. More specifically, I show how, by virtue of specific ritual processes, the nganga becomes the objectification of a set of relationships which constitute its owner's ritual identity such as the initiatory relationship, for example. Further on, by focusing on everyday life, I show that the nganga restructures the domestic settlements and modifies the palero's relation to her or himself. I argue that the nganga's replacement or destruction would challenge the palero's existence in a radical way.

\section{MOTS CLÉS}

Palo monte, Cuba, religions afro-cubaines, nganga, nfumbi, matérialité, rituel, vie quotidienne.

\section{KEYWORDS}

Palo monte, Cuba, Afro-Cuban religion, nganga, nfumbi, materiality, ritual, everyday life. 